University of New Hampshire

University of New Hampshire Scholars' Repository

9-23-2009

\title{
Advanced characterization and simulation of SONNE: a fast neutron spectrometer for Solar Probe Plus
}

\author{
R S. Woolf \\ University of New Hampshire - Main Campus \\ James M. Ryan \\ University of New Hampshire, James.Ryan@unh.edu \\ Peter F. Bloser \\ University of New Hampshire, Peter.Bloser@unh.edu \\ U Bravar \\ University of New Hampshire - Main Campus \\ E O. Fluckiger \\ University of Bern
}

See next page for additional authors

Follow this and additional works at: https://scholars.unh.edu/ssc

Part of the Astrophysics and Astronomy Commons

\section{Recommended Citation \\ Richard S. Woolf ; James M. Ryan ; Peter F. Bloser ; Ulisse Bravar ; Erwin O. Flückiger ; Jason S. Legere ; Alexander MacKinnon ; Procheta C. Mallik ; Mark L. McConnell and Benoît Pirard "Advanced characterization and simulation of SONNE: a fast neutron spectrometer for Solar Probe Plus", Proc. SPIE 7438, Solar Physics and Space Weather Instrumentation III, 74380S (September 23, 2009); doi:10.1117/ 12.826425; http://dx.doi.org/10.1117/12.826425}

This Conference Proceeding is brought to you for free and open access by the Institute for the Study of Earth, Oceans, and Space (EOS) at University of New Hampshire Scholars' Repository. It has been accepted for inclusion in Space Science Center by an authorized administrator of University of New Hampshire Scholars' Repository. For more information, please contact Scholarly.Communication@unh.edu. 


\section{Authors}

R S. Woolf, James M. Ryan, Peter F. Bloser, U Bravar, E O. Fluckiger, Jason S. Legere, A L. MacKinnon, Procheta Mallik, Mark L. McConnell, and B Pirard 


\title{
Advanced Characterization and Simulation of SONNE: a Fast Neutron Spectrometer for Solar Probe Plus
}

\author{
Richard S. Woolf*a, James M. Ryan ${ }^{\mathrm{a}}$, Peter F. Bloser ${ }^{\mathrm{a}}$, Ulisse Bravar ${ }^{\mathrm{a}}$, Erwin O. Flückiger ${ }^{\mathrm{b}}$, Jason S. \\ Legere $^{\mathrm{a}}$, Alexander MacKinnon ${ }^{\mathrm{c}}$, Procheta C. Mallik ${ }^{\mathrm{c}}$, Mark L. McConnell ${ }^{\mathrm{a}}$, Benoît Pirard ${ }^{\mathrm{d}}$ \\ ${ }^{a}$ University of New Hampshire, Space Science Center, Durham, NH, USA \\ ${ }^{b}$ University of Bern, Physikalisches Institut, Bern, Switzerland \\ ${ }^{c}$ University of Glasgow, Dept. of Physics and Astronomy, Glasgow, Scotland, UK \\ ${ }^{\mathrm{d} C A N B E R R A,} 67834$ Lingolsheim Cedex, France
}

\begin{abstract}
SONNE, the SOlar NeutroN Experiment proposed for Solar Probe Plus, is designed to measure solar neutrons from 1-20 $\mathrm{MeV}$ and solar gammas from $0.5-10 \mathrm{MeV}$. SONNE is a double scatter instrument that employs imaging to maximize its signal-to-noise ratio by rejecting neutral particles from non-solar directions. Under the assumption of quiescent or episodic small-flare activity, one can constrain the energy content and power dissipation by fast ions in the low corona. Although the spectrum of protons and ions produced by nanoflaring activity is unknown, we estimate the signal in neutrons and $\gamma$-rays that would be present within thirty solar radii, constrained by earlier measurements at 1 AU. Laboratory results and simulations will be presented illustrating the instrument sensitivity and resolving power.
\end{abstract}

Keywords: Solar Probe Plus, Solar Neutrons, Nano-flaring, Neutron Detection, Neutron Simulations

\section{INTRODUCTION}

\subsection{Solar Probe Plus Mission}

One of the perplexing and still unanswered questions in solar physics is: what is the cause of the million degree solar corona? To help solve this problem, a mission to the inner heliosphere to make in situ measurements in the low corona is needed. This original motivation for this mission has been expanded to neutron measurements in order to achieve a greater understanding of energetic ions in the corona. Our current knowledge is limited by the sensitivity and threshold to $\gamma$-ray lines that have been measured from $1 \mathrm{AU}$; a neutron spectrometer with $\gamma$-ray detection capabilities would achieve a lower threshold and a gain in the sensitivity. Currently, only a fraction of the full picture of the Sun is understood from neutron and $\gamma$-ray measurements made on previous missions from large (bright) events. More sensitive measurements will allow for a greater observational database, and would enhance the current understanding of the processes in the low corona. A neutron/ $\gamma$-ray spectrometer at Solar Probe Plus distances would be sensitive to smaller and lower energy events, which should help to decipher the role that low energy ions play in these events. Such missions, while ambitious, were thought to be impossible given the harsh near Sun environment and the consequent cost and technology. The idea of Solar Probe was initially to rendezvous within 4 solar radii of the Sun in a single pass mission. The mission was reworked and modified in 2008 into a new concept - Solar Probe Plus. Now, with a perihelion of 9.5 solar radii, the limits on spacecraft mass and power budget have since been relaxed, and the orbit is such that the spacecraft can make multiple passes with more observation time within 30 solar radii. The mission is slated for a launch in 2015, but may be as late as 2018 [1]. The spacecraft would reach perihelion 6.9-7.7 years later, depending on the number of Venus gravity assists needed for its final orbit.

*rwoolf@unh.edu; phone 1603 862-4595; fax 1603 862-3584;

Solar Physics and Space Weather Instrumentation III, edited by Silvano Fineschi, Judy A. Fennelly, Proc. of SPIE Vol. 7438, 74380S · (c) 2009 SPIE · CCC code: 0277-786X/09/\$18 - doi: 10.1117/12.826425 
Perihelion is scheduled to coincide with the expected maximum of Solar Cycle 25. A neutron $/ \gamma$-ray spectrometer has been commissioned to be on-board the Solar Probe Plus mission. We will describe the laboratory and simulation studies that have been performed for a proposed instrument for this mission known as SONNE: the SOlar NeutroN Experiment.

\subsection{Solar Neutrons}

Neutrons are produced over a wide range of energies when flare accelerated protons and ions interact with the ambient solar atmosphere. The closest we can come to an unbiased measurement of the energetic proton spectrum at the flare site comes from this secondary neutral radiation - neutrons and $\gamma$ rays - the parent of which is the accelerated proton spectrum at the flare site. They retain a significant fraction of the incident proton or ion energy and leave the corona unaffected by transient magnetic fields, escaping into interplanetary space. However, the low-energy spectrum of solar neutrons has not been measured due to the neutron lifetime of $\sim 887 \mathrm{~s}$. Neutrons $\beta$ decay into a proton, electron, and anti-electron neutrino. Another competing factor is flux divergence, or $\mathrm{r}^{-2}$ effects, which will also contribute to the reduction in the numbers of low-energy neutrons at $1 \mathrm{AU}$ (Figure 1). Both of these consequences combine to make low energy neutron observations at $1 \mathrm{AU}$ impossible. Thus, proximity to the Sun to perform these measurements is essential.

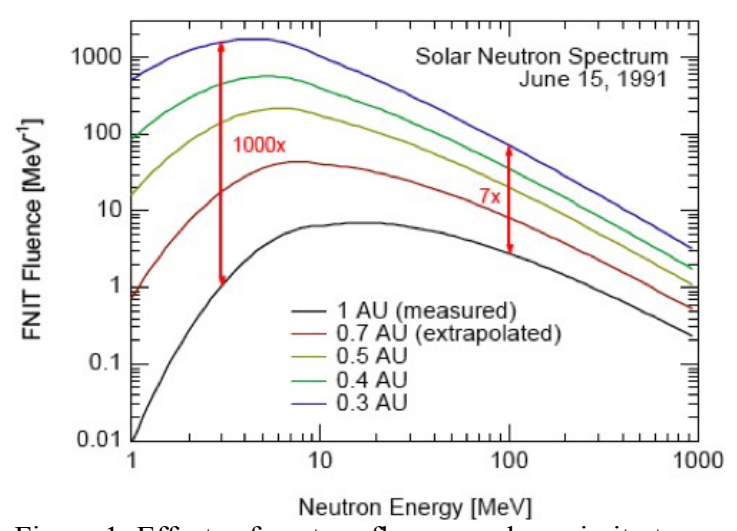

Figure 1: Effects of neutron fluence and proximity to the Sun for the 15 June 1991 X-12 flare.

Simulations show that flare accelerated particles with a soft energy spectrum interacting with a heavy ion-rich target would produce a large yield of neutrons in the $1-20 \mathrm{MeV}$ range [2]. Detection and measurement of these low energy neutrons would provide key spectral, compositional and temporal information along with insight into the acceleration mechanism that energizes particles on short timescales during a solar flare. The electromagnetic processes responsible for the energization of protons, ions and electrons must depend on the charge/mass ratio of the particle, its momentum and the manner that it interacts with hydromagnetic waves. Furthermore, how particles are selected and pre-energized remains a lingering problem, because the injection processes biases the intensity, composition and spectrum of the accelerated particles [3]. For maximum diagnostic capabilities, a fast neutron spectrometer working in tandem with a $\gamma$ ray spectrometer would provide excellent and complementary measures of the accelerated ion spectrum along with the target composition. The shape of the neutron and $\gamma$-ray production cross sections differs for increasing incident projectile energy. The $\gamma$-ray cross section varies - large at low energies and then sharply decreasing at higher energies - while the neutron cross-section increases abruptly at low energies and remains steady for energies up to several hundred $\mathrm{MeV}$ nucleon $^{-1}$, as shown in Figure 2. Sampling the production of both neutrons and $\gamma$-rays over a wide range of cross sections is needed to provide overlapping measurements for the complete understanding of the composition and processes in the corona. It is thus important to quantify the variability of particle acceleration on all fronts. This has been recognized as an important goal by the solar physics community, and is to be addressed by the Solar Probe Plus mission, or similar mission

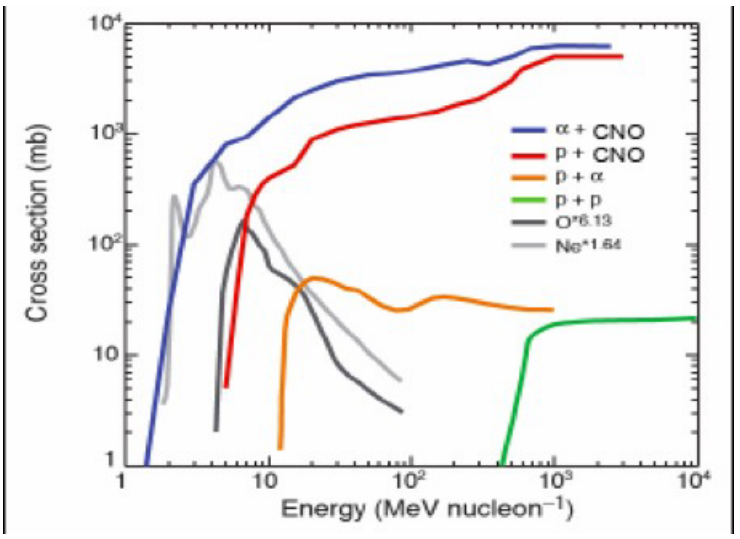

Figure 2: Key neutron production cross sections concepts [4] [5]. 


\subsection{Quiescent Solar Neutrons}

A possible agent for heating of the solar corona is a steady-state or quasi-continuous particle acceleration at the Sun. At Solar Probe Plus distances, SONNE has the potential to reveal evidence of proton and ion acceleration in the low corona and could lead to the discovery of micro- and nano-flaring activity during quiet sun times and help explain the coronal heating problem. Such small flares could accelerate short bursts of $\mathrm{MeV}$ ions that range out in the corona or escape into space. As such, they would serve to heat the corona via non-thermal dissipation of energy and/or provide a steady stream of seed particles into the interplanetary medium. Their presence could be detectable through quasi-steady low-energy neutron emission, where alphas and heavier accelerated ions would release neutrons after collisions in the low corona or photosphere. Alphas and heavier nuclei are far more prolific at low energies in producing neutrons (Figure 2). Impulsive-flare compositions are known to be rich in heavier nuclei. The neutron spectra will be used to search for quiescent neutron emission from the Sun and to measure the number of low and medium energy ions interacting during a solar flare. These data will be combined with the $\gamma$-ray data to arrive at an energy spectrum and composition profile for the energetic particles generated by a flare. The possibility of numerous small flares heating the corona [6] cannot be supported or refuted with data from $1 \mathrm{AU}$. While there is evidence for micro-flares in the hard X-ray data from GRS on SMM [7] and the UV energy band [8], no such evidence in neutrons or $\gamma$-rays has been found thus far. Previous efforts [9] were completed with COMPTEL on the Compton Gamma-Ray Observatory (CGRO) to search for the 1-10 MeV $\gamma$ ray nuclear line emission during times of quiet Sun. The results have been used to place a $2 \sigma$ upper limit of the quiescent $\gamma$-ray flux of $2.1 \times 10^{-5} \gamma \mathrm{s}^{-1} \mathrm{MeV}^{-1}$. Proximity to the Sun will also aid in SONNE $\gamma$-ray detection sensitivity of weak flares and the $\gamma$-ray flare emission that could present itself during times of quiescence. Since Solar Probe Plus will be spending more time than originally planned within thirty solar radii, it will be able to observe the Sun over a large fraction of the total solar cycle. This will allow observation of the variation of flux measurements for varying levels of solar activity.

\section{SONNE - SOlar NeutroN Experiment}

\subsection{Measurement Principles}

To achieve the necessary sensitivity one needs an instrument that measures the full energy of the neutron/ $\gamma$-ray and rejects background from directions other than the Sun. A double-scatter telescope is such an instrument. Because neutrons are electrically neutral, neutrons must be detected using indirect means. The preferred method at $\mathrm{MeV}$ energies exploits the large n-p elastic scatter cross-section (orders of magnitude larger than inelastic). The ideal material, acting both as neutron scatterer and recoil proton detector, is an organic scintillator. Neutrons that undergo two scatters have their energy uniquely determined by measuring the coordinates, relative time and recoil proton energy of every elastic n-p interaction. These data are analyzed on an event-by-event basis using n-p scattering kinematics to determine each incident neutron energy and scattering angle. The preferred method for $\gamma$-ray detection in the $1-10 \mathrm{MeV}$ range is Compton scattering. For the first scatter the incident $\gamma$-ray will Compton scatter in a low- $Z$ material and produce a measureable recoil electron. The scattered $\gamma$-ray will then deposit its remaining energy in the second scatter in a high-Z material such as $\mathrm{NaI}(\mathrm{Tl})$ or $\mathrm{LaBr}_{3}$. Using the principles of Compton kinematics, the full energy of the $\gamma$-ray is reconstructed from the sum of the energy deposits in the first and second scatter. Neutrons/ $\gamma$-rays that undergo at least two scatters and make a full energy deposit have their incident velocity vector constrained along the mantle of a cone as in Figure 3. The half angle of the cone $(\phi)$ is given by:

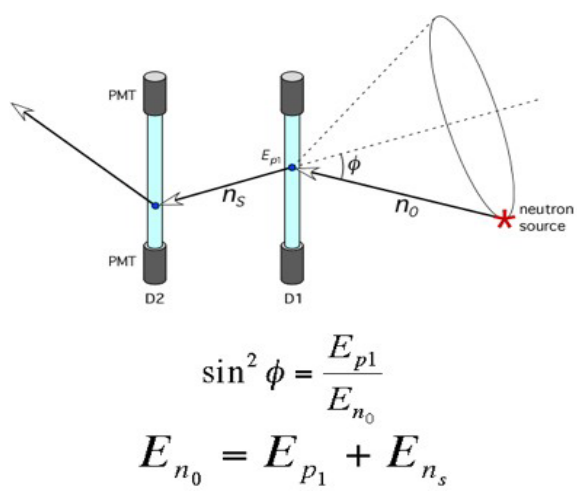

Figure 3: Depiction of double n-p scatter 


$$
\sin ^{2} \phi=\frac{E_{p 1}}{E_{n}} .
$$

The size and direction of this "event cone" is determined from accurate energy and position measurements. To perform imaging, the event cone is then projected onto an image plane, where it intersects to become an "event circle." Each point on the event circle is a probabilistic origin direction of the incident neutron. The highest density of intersections statistically determines that direction. This procedure is analogous to that used successfully by the NASA space-based COMPTEL instrument to image extragalactic $\gamma$-rays and local neutron sources [10]. Figure 4 shows that principle demonstrated with the superposition $\sim 100 \gamma$-ray events on the celestial sphere from GRB910503. Figure 5 shows a neutron image of the Sun as seen with $\sim 200$ neutrons in the 15-80 MeV energy range produced during the 15 June 1991 flare.

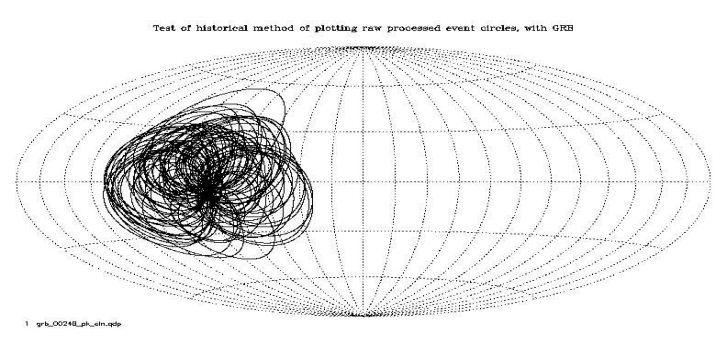

Figure 4: $\gamma$-ray event circles from GRB910503 measured with COMPTEL

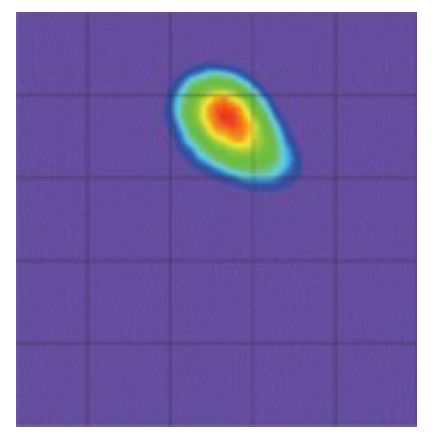

Figure 5: Image of the Sun in $15-80 \mathrm{MeV}$ neutrons from the 15 June 1991 X-12 flare.

\subsection{Spacecraft Instrument Description}

The proposed instrument for the Solar Probe Plus mission must be compact, lightweight and run on low power. The design outlined for the neutron detector is based on a previous trade study performed with FNIT - a Fast Neutron Imaging Telescope. The prototype of this instrument employs cylindrical rods filled with liquid scintillator for neutron detection and measurement. The advantage of a liquid scintillator detector is its ability to perform pulse shape discrimination (PSD), which increases the instrument sensitivity. PSD allows for the discrimination between $\gamma$-rays and neutrons based on the shape of the decaying tail pulse. The performance of the FNIT prototype demonstrated a neutron energy resolution of $20 \%(\sigma)$ and an angular resolution of $5^{\circ}(\sigma)$ [11]. However, an instrument of this size and mass would be impractical for a mission to the inner heliosphere. A scaled down version of this instrument - SONNE - is envisioned as: two small cylindrical cells each filled with liquid scintillator and with a photomultiplier tube (PMT) coupled to one end. The cell in the first detection plane (D1) is composed of organic liquid scintillator, e.g. BC-519. The cell in the second detection plane (D2) is also organic liquid scintillator. However, the two liquids differ in their hydrogen content and therefore in the light output; D2 contains more hydrogen, making the cell more efficient for detecting n-p scatters. However, the lower light output, compared to that of BC-501, degrades the energy and angular resolution performances. For $\gamma$-ray sensitivity a third detection plane (D3) of $\mathrm{LaBr}_{3}$ and a PMT coupled to it was added. $\mathrm{LaBr}_{3}$ is a dense high-Z material with good stopping power of $\gamma$-rays, allowing for total energy absorption. $\mathrm{LaBr}_{3}$ also has a high light output yielding excellent energy resolution (of the order 3\%.) The separation between the elements is 12 $\mathrm{cm}$ center-to-center on-axis.

Solar Probe Plus SONNE is designed to conform to the strict mass $(4 \mathrm{~kg})$ and power budgets $(4 \mathrm{~W})$. For the given volume restrictions of the instrument, the D1/D2 cells would have a radius of $3.81 \mathrm{~cm}$ with a thickness of $3 \mathrm{~cm}$; each cell would be contained in magnesium housing with $0.1 \mathrm{~cm}$ thick walls on the top and bottom with $3 \mathrm{~cm}$ on the side. The PMT would be housed in $0.1 \mathrm{~cm}$ aluminum. The D3 cell would be have a thickness of $3 \mathrm{~cm}$ with a radius of $3.81 \mathrm{~cm}$. 
The crystal would be housed in $0.05 \mathrm{~cm}$ thick aluminum walls. Data for the double scatter instrument would be acquired in the following format of: pulse heights, pulse shapes, and time-of-flight. Each double-scatter event should generate approximately 128 bits of data. With minimal data storage available on-board and a limited telemetry rate of $\sim 100 \mathrm{bits} / \mathrm{s}$, carrying out the process of de-convolution - deducing the true neutron distribution - may prove to be desirable. Several de-convolution methods have been successfully demonstrated for neutron measurements in space, such as Tikhonov and Singular Value Decomposition. Using these methods to reduce the acquired data open up the possibilities for various degrees of data compression [12].

\subsection{Prototype Description}

A prototype that closely mimics the design outlined for the mission has been built and tested at the University of New Hampshire. It consists of a three-layer detection system - the D1 and D2 layers are liquid scintillators cells, each with a diameter of 1.5 ". The third layer is a $2 " \times 2 " \mathrm{NaI}(\mathrm{Tl})$ crystal, which was used due to the availability and cost considerations. Custom made preamplifiers produce a negative, fast signal that is read out to a Mesytec MPD-4 module [13]. The output of the MPD-4 was used for pulse height measurements, time-of-flight, coincidence trigger, and when applicable, PSD. The MPD-4 internal settings have been adjusted via a terminal window and USB connection. Adjustable settings are: gain, threshold, neutron discrimination and walk. The latter are used for fine-tuning the PSD circuit.

The prototype pulse height and shape information were calibrated and used for energy, time-of-flight, and PSD. We assumed that the interaction takes place at the center of each scintillator cell since there is no position output available. The light output function was determined over the full dynamic range by using $\gamma$-ray emitting isotopes. These were chosen due to the monoenergetic nature and that they interact primarily with electrons in the scintillator material. The amount of light that they produce increases linearly with increasing energy and hence useful for conversion between measured light output (pulse height) and electron equivalent energy. Isotopes with energies ranging from $22 \mathrm{keV}$ to 1275 $\mathrm{keV}$ were used to calibrate each detector; this allowed for determination of threshold and dynamic range. The dynamic electron equivalent energy range (and threshold) of $\sim 10 \mathrm{keV}-2 \mathrm{MeV}$ was found for D1/D2 and $\sim 300 \mathrm{keV}-2 \mathrm{MeV}$ for D3. Neutrons can deposit their energy through a variety of interactions; elastic scattering between neutrons and protons has the largest cross section, over the competing reactions involving inelastic $n-p$ scattering and $n-C$ scatters, in the 1-10 $\mathrm{MeV}$ range. The energy the neutron imparts to the recoil protons was used as part of the process for reconstructing the incident energy. Therefore a conversion between the electron equivalent energy and the proton equivalent energy was needed; several references for this conversion are available elsewhere [14] [15]. The performance of D1/D2 (in terms of pulse height resolution) was $20 \%(\sigma)$ at $60 \mathrm{keV}$ and improving to $10 \%(\sigma)$ at $374 \mathrm{keV}$. For the $\mathrm{Na}(\mathrm{Tl})$, or D3, a measured energy resolution of $10 \%$ (FWHM) is found at $662 \mathrm{keV}$.

As mentioned above, a Mesytec MPD-4 module was used for the measurement of pulse shape and for the time-of-flight trigger. For timing, the module uses the Constant Fraction Discriminator (CFD) for time pickoff. The fast pulse from each module was used for the START and (delayed) STOP inputs for separate Time-to-Amplitude Converters (TACs). The coincidence resolving time of the prototype was determined with a ${ }^{60} \mathrm{Co}$ source, which emits simultaneous $\gamma$-rays in random directions when $\beta$-decaying into stable ${ }^{60} \mathrm{Ni}$, placed equidistant between the two detecting modules in the coincidence mode. A coincidence resolving time (CRT) of $0.98 \mathrm{~ns}(\sigma)$ was measured. The module performs PSD by taking the ratio of the fast-to-slow input signal [16], which was read out directly from the TAC output on the MPD-4 to an Analog-to-Digital Converter (ADC) for processing. An Americium-Beryllium (Am-Be) neutron/ $\gamma$-ray source was used to tune the MPD-4 PSD settings to obtain the best figure of merit, which is a measure of the separation that can be achieved between different types of evens in a given application [17].

\subsubsection{Prototype Performance}

A significant fraction of energetic ions accelerated during times of nano-flares would produce an ion spectrum with a $\sim 1$ $\mathrm{MeV}$ exponential cutoff. The dominant reaction channel responsible for the neutron emission at these low energies is $\alpha-$ 
particles inelastic scattering off elements heavier than helium. The neutron spectrum would therefore be evaporation-like near threshold for production, i.e., an exponential with an upper limit of $10 \mathrm{MeV}$. The complete energy spectrum of these neutrons emitted from small flares is, however, unknown; the final shape could be affected by a varying medium in the low corona that these neutrons must pass through before measurement. Nevertheless, the SONNE prototype was tested with a source of ${ }^{252} \mathrm{Cf}$ fission neutrons, producing an evaporation-like spectrum up to $\sim 10 \mathrm{MeV}$. Decay by alpha emission (96.91\% probability) and spontaneous fission (3.09\% probability) result in a half-life of 2.6 years and neutron emission of $2.314 \times 10^{6} \mathrm{n} \mathrm{s}^{-1} \mu \mathrm{g}^{-1}$ with a specific activity of $0.536 \mathrm{mCi} \mu \mathrm{g}^{-1}$. The neutron spectrum is similar to a fission reactor with most probably energy of $0.7 \mathrm{MeV}$ and average energy of $2.1 \mathrm{MeV}$ [18]. The $\gamma$-ray distribution closely resembles an evaporation spectrum, with $80 \%$ of the photons released upon each fission having energy of less than 1 $\mathrm{MeV}$ [19]. The source that was used by the University of New Hampshire has a mass of $5.1 \mu \mathrm{g}$ and an activity of 2.6 $\mathrm{mCi}$, which yields $\sim 15 \times 10^{6}$ neutrons s $\mathrm{s}^{-1}$ and $\sim 20 \times 10^{6} \mathrm{\gamma} \mathrm{s}^{-1}$. The energy reconstruction, angular resolution and imaging performance of the prototype were tested with the ${ }^{252} \mathrm{Cf}$ and a ${ }^{137} \mathrm{Cs}$ isotope.

\subsubsection{Angular Resolution}

The angular resolution measurement (ARM) of the prototype was determined by taking the difference between the calculated scattering angle $\left(\phi_{\mathrm{n}}\right)$ and the geometric scattering angle $\left(\phi_{\mathrm{g}}\right)$. The calculated scattering angle is obtained from the relation given in equation 1, while geometric scattering angle, using the true scatter angles calculated from the measured positions of the detectors. For ${ }^{252} \mathrm{Cf}$ at $3 \mathrm{~m}$, an angular resolution of $5^{\circ}(\sigma)$ was measured as shown in Figure 6. The angular resolution for $\gamma$-ray measurement was determined by using a strong ${ }^{137} \mathrm{Cs}$ source $(662 \mathrm{keV})$ placed at a distance of $3 \mathrm{~m}$. The full energy spectrum for $\gamma$-ray events was found by simply adding the pulse height in either D1 or $\mathrm{D} 2$ to that in D3. Fully absorbed events were defined as those lying within the $662 \mathrm{keV}$ photopeak. Using these events, the ARM distribution (Figure 7) was found for both D1-D3 (dotted line) and D2-D3 (solid line) scatters. The widths of these distributions were found to be $3.2^{\circ}(\sigma)$ for D1-D3 and $4.1^{\circ}(\sigma)$ for D2-D3.

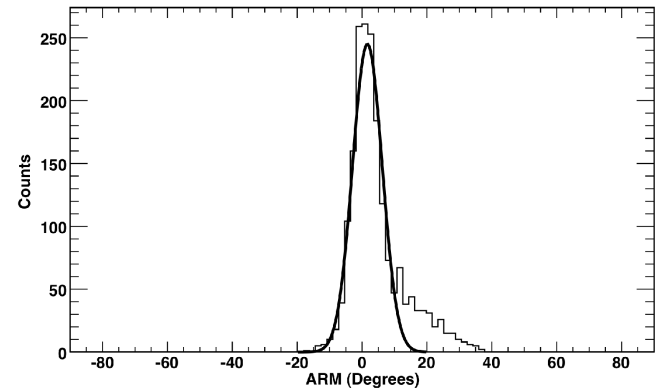

Figure 6: ARM distribution for fission neutrons. The darker line is a Gaussian fit applied to $\pm 20^{\circ}$ from center.

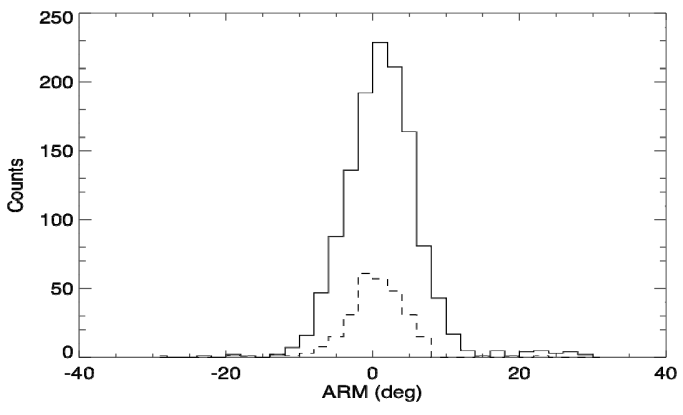

Figure 7: ARM distribution for ${ }^{137} \mathrm{Cs} \quad \gamma$-rays for interactions occurring between D1-D3 and D2-D3.

\subsubsection{Energy Reconstruction}

The incident energy spectrum is reconstructed on an event-by-event basis. Each event trigger is subject to selection criteria based on pulse height and shape, time-of-flight, and threshold. If satisfied for neutrons, then conversion of the pulse height to proton equivalent energy and time-of-flight selections are applied to yield physical quantities. Nonrelativistic scattering kinematics are used to reconstruct the total incident energy of each neutron and to build up the full energy spectrum. Performing this task yields a count spectrum - energy measurements uncorrected for instrumental effects. Figure 8 shows the results for the SONNE prototype viewing the ${ }^{252} \mathrm{Cf}$ source from a distance of $3 \mathrm{~m}$. The fission $\gamma$-ray spectrum is reconstructed in Figure 9. To select all fully absorbed $\gamma$-ray events, ARM selection criterion lying within $\pm \mathrm{FWHM}$ of $0^{\circ}$ of photopeak was used to reconstruct the spectrum. 


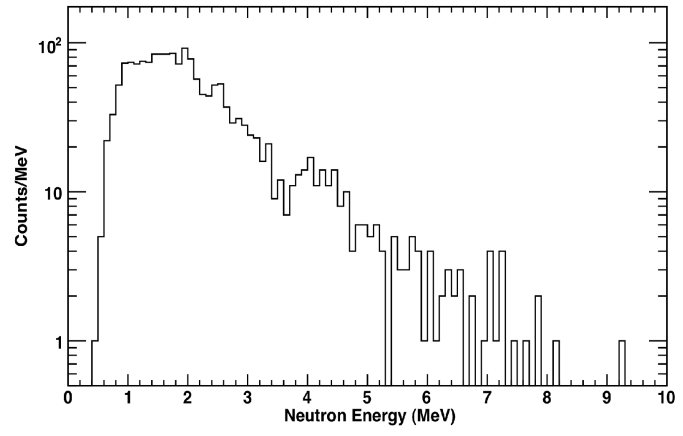

Figure 8: Reconstructed fission neuron count spectrum

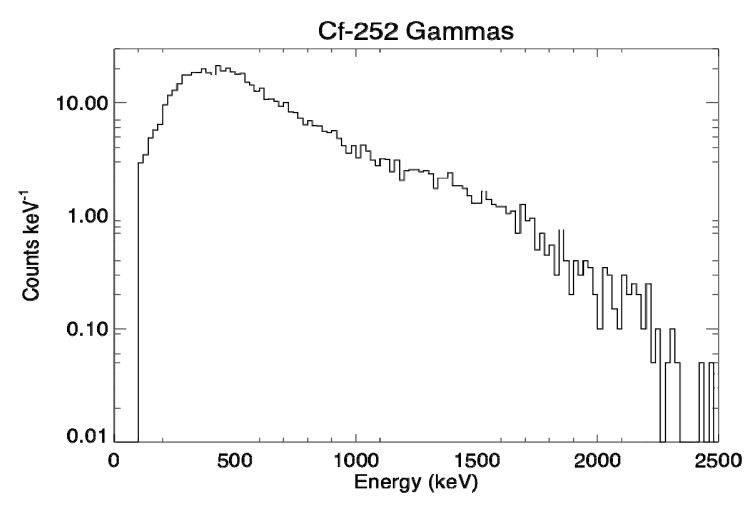

Figure 9: Reconstructed fission $\gamma$-ray count spectrum

\subsubsection{Neutron Imaging}

First order imaging principles - not employing the instrument response or the point spread function — were developed to back project neutron event circles onto an image plane. This was done for proof of concept for near-field applications; space-based imaging would map event circles onto the celestial sphere via three coordinates - scattering angle, and the altitude and azimuth angle. For this application neutron event circles are produced using direction cosines and normalized unit vectors for the known distance of the image plane. Euler rotation angles are then applied for rotation of the event circles such that they will intersect the image plane based on the incident angle. A set of 360 points - one for each degree of the circle - are plotted on the image plane to create an unbiased probability distribution of possible locations for the source. The image plane is segmented into small-scale bins; each point of the circle that crosses the bin boundary is incremented. A 3-d histogram is used to count the number of intersections in each bin - the most intersections correspond to most probable source location. Figure 10 demonstrates the imaging principle with the neutron data from ${ }^{252} \mathrm{Cf}$ at $3 \mathrm{~m}$ and $\pm 30^{\circ}$. The angular resolution of the instrument directly affects its imaging capabilities. Errors in the event circle annulus width arise due to inaccuracy in the energy reconstruction, which depends on pulse height measurement and energy conversion, interaction location, and time-of-flight measurement. Projection of the event circle on the image plane is affected by the position measurement. However, one can see that choosing forward scatter events and using the principles of imaging can also achieve directionality.

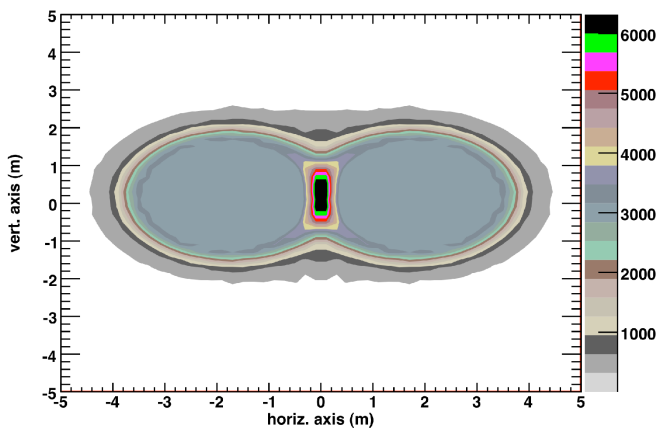

Figure 10: Intensity contours of event circle intersections for ${ }^{252} \mathrm{Cf}$ viewed at $\pm 30^{\circ}$ from a distance of $3 \mathrm{~m}$. Source is located at $(0,0)$ in the image plane.

\section{Simulations}

\subsection{Neutron Simulations}

Monte Carlo calculations were performed to assess the sensitivity of SONNE in the environment on-board the Solar Probe Plus spacecraft. For neutron simulations, GEANT4 (v9.1.p03) - a simulation package designed to track and record the interaction of incident particles and/or photons as they pass through matter - was used as the platform to construct a mass model of the instrument and spacecraft [20]. The SONNE instrument was exposed to neutron fluence indicative of a X-12 flare observed from various distances in the inner heliosphere. The neutron fluence was extrapolated to distances 
of 10, 20, and 30 solar radii based on the 15 June 1991 neutron fluence as measured by COMPTEL on the CGRO at 1 AU. The source of background used is the secondary neutron flux produced from Galactic Cosmic Rays (GCRs) consisting of high-energy protons - interacting with the spacecraft material. Computing the signal-to-noise ratio for a given measurement yields the sensitivity of the instrument for specific conditions of neutron and background flux.

\subsubsection{Energy Reconstruction \& Spacecraft Induced Background}

In June of 1991 a large class of X-flares erupted from the Sun during a two-week period. One of these events, occurring on the 15 June, produced an $\mathrm{X}-12 / 3 \mathrm{~B}$ class flare that was well documented by the CGRO. Neutrons in the $15-80 \mathrm{MeV}$ energy range were detected and measured for this event from a distance of $1 \mathrm{AU}$. The time integrated directional neutron emissivity spectrum was derived from the number of detected counts and was found to be $8.8 \times 10^{27} \mathrm{n} \mathrm{sr}^{-1}$ and a spectral index $\mathrm{s}=1.5$ [21]. This information was then used to predict the neutron fluence below $15 \mathrm{MeV}$ and at different heliocentric distances. Figure 11 shows the results of the solar neutron fluence spectrum at 10,20, and 30 solar radii. The intensity of $1 \mathrm{MeV}$ neutrons at Solar Probe Plus perihelion is $\sim 9$ orders of magnitude greater than it is at $1 \mathrm{AU}$, and $\sim 5$ orders of magnitude greater for $10 \mathrm{MeV}$ neutrons [22]. Each flares spectra shown in Figure 11 was used to compute the SONNE response for a $2100 \mathrm{~s}$ observation time.

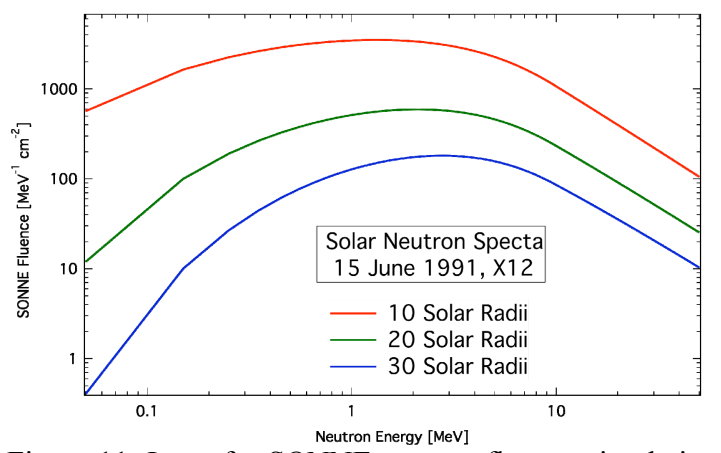

Figure 11: Input for SONNE neutron fluence simulations. $\mathrm{X}-12$ flare of 15 June 1991 as seen from 10, 20, and 30 solar radii

Additionally, the SONNE sensitivity is crucial for assessing the instrument performance. To determine the sensitivity, background neutron events are added into the measurement window. A mass model of the Solar Probe Plus spacecraft was built into the simulation code based on the specifications given by the Solar Probe Plus Science and Definition Team Document [23]. The spacecraft consists of a heat shield (mass: $70 \mathrm{~kg}$, composition: $62 \% \mathrm{C}, 38 \% \mathrm{Al}$ ), spacecraft body (mass: $313 \mathrm{~kg}$, composition: $41 \% \mathrm{Al}, 13 \% \mathrm{~N}, 12 \% \mathrm{Cu}, 8 \% \mathrm{Si}, \ldots$ ), solar arrays (mass: $100 \mathrm{~kg}$, composition: TBD), and hydrazine fuel tanks (mass: $50 \mathrm{~kg}$, composition: $87 \% \mathrm{~N}, 13 \% \mathrm{H}$ ). To replicate the local spacecraft background that would be present in the harsh radiation environment of deep space, a proton-only, omni-directional cosmic ray flux $(\alpha-$ particles and heavier ions were neglected) was used. The interplanetary field and solar wind in the heliosphere affect the total flux and spectrum of the galactic cosmic rays. This is not constant and creates a variable spectrum of galactic cosmic rays throughout the heliosphere. The variation of the modulation is related to the variation of the solar activity, which is commonly described by the so-called force field model [24]. The only model parameter is the modulation potential, $\phi$, given in units of [MV]. For conditions of solar minimum $(\phi=400 \mathrm{MV})$, this will model was used to generate a differential GCR spectrum in the inner heliosphere ranging from $10 \mathrm{MeV}-100 \mathrm{GeV}$. Figure 12 shows the secondary background neutrons that are produced from GCRs interacting with the spacecraft.

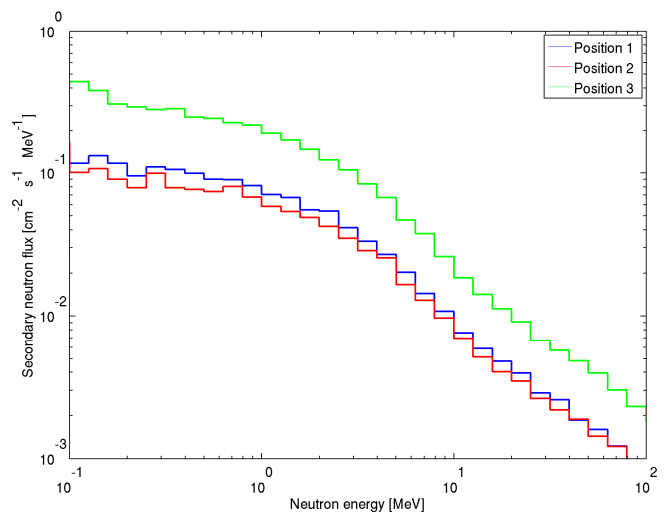

Figure 12: Secondary background neutron flux for three possible SONNE locations on-board Solar Probe Plus. 


\subsubsection{Post Processing Algorithms \& Sensitivity}

The data generated from the simulation are then further analyzed in post processing [25]. Double scatter events are artificially broadened based on measured laboratory resolutions and Gaussian statistics for time-of-flight, position of interaction, and energy deposited. The number of a multiple scatters in each cell, $\mathrm{n}-\mathrm{C}$ scatters, and inelastic scatters are tracked and recorded for later analysis of the instrument response. This smoothing of the data is performed to replicate a more realistic instrument. Figure 13 shows the differential response of SONNE at the various heliocentric distances. The black curve is the background neutron counts integrated over the $2100 \mathrm{~s}$ observation time that fall within the $\pm 20^{\circ}$ ARM cuts of the solar direction. The sensitivity of the SONNE instrument is given in terms of the signal-to-noise ratio, expressed as the signal (S) divided by the square root of the background (B). For an energy range of $1-20 \mathrm{MeV}$, at perihelion a detection significance of $40 \sigma$ is obtained for the given neutron flux and by suppressing the background using imaging.

For comparison, we computed the sensitivity of the Gamma Ray and Neutron Spectrometer (GRNS) on-board the Mercury MESSENGER mission. The NS is a $1 \mathrm{~kg}$ (3.9 $\mathrm{kg}$ total) bulk scintillator detector that will be used to map variations in neutrons that Mercury's surface emits when struck by cosmic rays [26]. Currently en route to the innermost planet on its way to final orbit in 2011, this is the first time a neutron detector has been at this proximity to the Sun. With a perihelion of 60 solar radii and comparable observation time to a X-12 flare, a non-imaging - therefore less sensitive instrument would find a detection significance of $13 \sigma$.

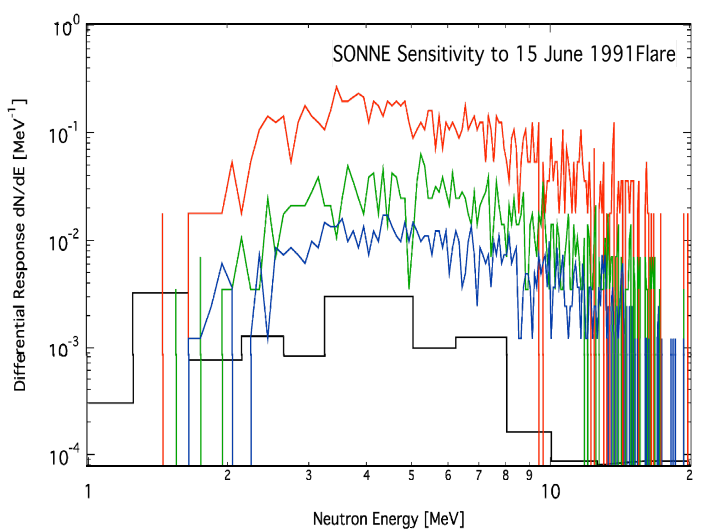

Figure 13: Reconstructed count spectra for 10 (red), 20 (green), and 30 (blue) solar radii. Black curve is the suppressed background using imaging

\section{$3.2 \gamma$-rays}

The $\gamma$-ray simulations for SONNE were performed with MGGPOD - a GEANT3 based Monte Carlo suite commonly used in $\gamma$-ray astronomy [27]. The performance of the SONNE instrument for detecting and reconstructing the energy spectra from solar flare $\gamma$-rays was tested. Shown in Figure 14 are the reconstructed spectra for interactions that happen between D1-D3, D2-D3, and single scatters in D3. Event selections were made on $\phi_{\mathrm{n}}$, the geometric scattering angle, and

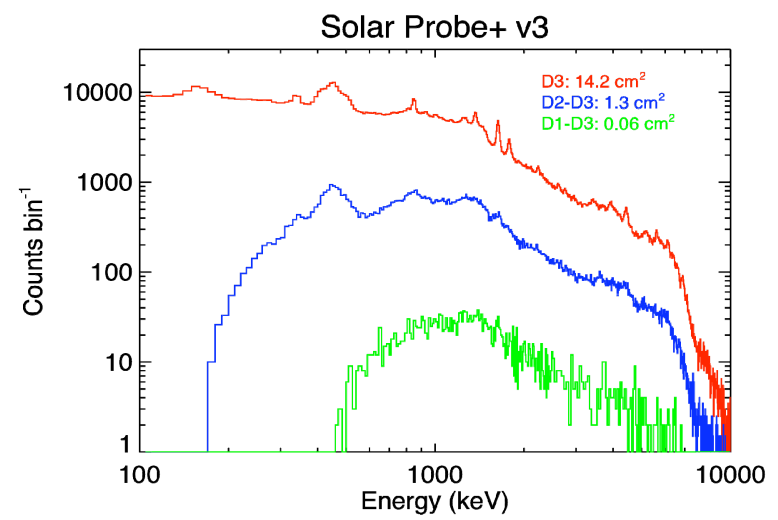

Figure 14: Reconstructed count spectra of solar $\gamma$-ray flare by D1-D3, D2-D3 double scatter events and D3 single scatters

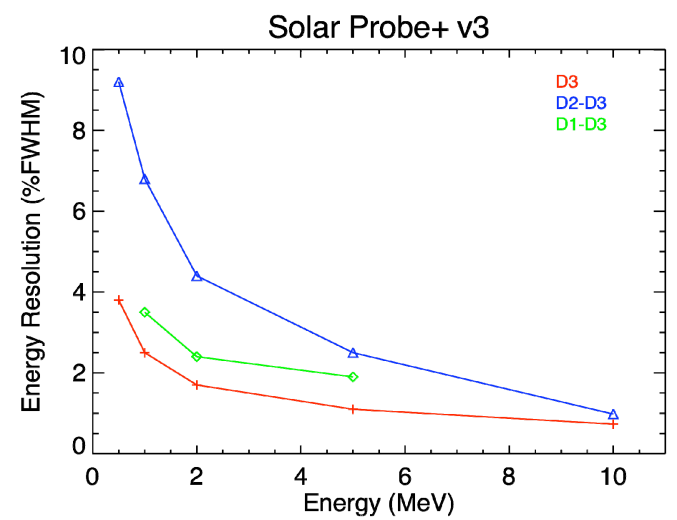

Figure 15: Energy resolutions (FWHM) for $\gamma$-ray measurements using D1-D3, D2-D3, and D3 singles. 
the ARM based on lab measurements. ARM cuts on $\pm 15^{\circ}$ and $\phi_{\mathrm{n}}$ cuts of $<27^{\circ}$ were made on D1-D3 scatters; ARM cuts on $\pm 45^{\circ}$ and $\phi_{\mathrm{n}}$ cuts of $<75^{\circ}$ were made on D2-D3 scatters. The effective area of the instrument is given as the weighted average over the full energy range of $0.1-10 \mathrm{MeV}$. D2-D3 double scatters have an effective area greater by two orders of magnitude over D1-D3 scatters. The energy resolution (Figure 15) is best for double scatters to measure the full energy of the incident $\gamma$-ray, important for resolving the narrow lines from nuclear emissions during flare activity. For the D1-D3 and D2-D3 curves, additional $\phi_{\mathrm{n}}$ cuts $\left(<30^{\circ}\right.$ for D1-D3, and $<50^{\circ}$ for D2-D3) have been added. While the D1-D3 $\phi_{\mathrm{n}}$ are limited by the geometry of the instrument, the D2-D3 events have a compromise between effective area and energy resolution: the larger the range of scatter angles allowed, the worse the energy resolution.

\subsection{Quiescent Solar Neutrons \& Coronal Heating}

A sensitivity estimate to quiescent solar neutrons was performed to determine what the measurable effects of coronal heating may be detected by SONNE in the inner heliosphere. The secondary neutron flux was subjected to the same ARM cuts that were applied to the flare data, only considering events that are within $\pm 20^{\circ}$ of the Sun direction. This restriction and selectivity severely reduces the count rate. For a two-week observation time - the approximate time within 20 solar radii - one would expect a total of 35 background neutron counts with the instrument at a position on the spacecraft where the background is least (blue curve in Figure 16). $3 \sigma$ detection significance would then correspond to an additional 20 counts. If Solar Probe Plus were to measure 20 background counts, the source rate would need to be $1.8 \times 10^{-5}$ counts $\mathrm{s}^{-1}$ yielding a source neutron emissivity at the Sun of $3.7 \times 10^{18}$ neutrons s${ }^{-1} \mathrm{sr}^{-1}$ given the surface area of both the Sun and the SONNE instrument. The effects of $\beta$-decay and flux divergence have been included. For this emissivity, it was then considered how many neutrons near threshold $-15 \mathrm{MeV}-$

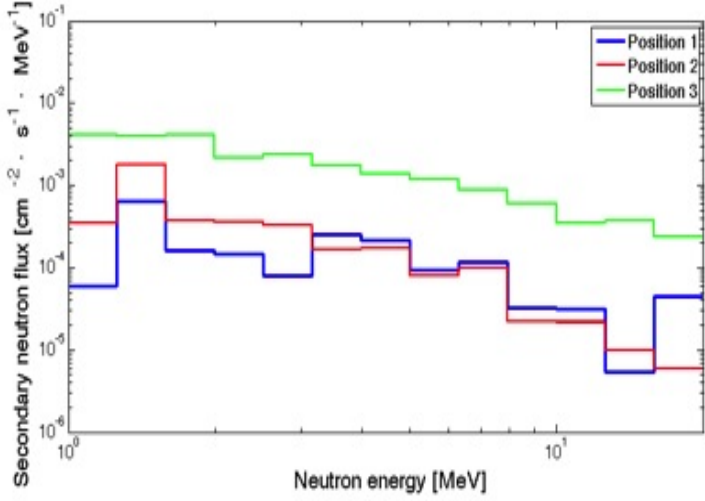

Figure 16: Secondary background neutron flux after imposing ARM cuts of $\pm 20^{\circ}$ of the Sun direction. Three positions correspond to the simulated potential location of the Solar Probe Plus spacecraft where SONNE could be located. For background calculations, position 1 (blue curve) was used. COMPTEL would have expected to observe over a twoweek period of quiescence. $\beta$-decay does not play as strong of a role for $15 \mathrm{MeV}$ neutrons as it did at $1 \mathrm{MeV}$, but flux divergence of these near threshold neutrons at $1 \mathrm{AU}$ is significant. Given an exponential-like neutron spectrum from small scale flaring and a neutron effective area of $\sim 5 \mathrm{~cm}^{2}$, COMPTEL would have expected to see of the order $\sim 10^{-9} \mathrm{n}$ $\mathrm{sr}^{-1}$. This emissivity, given the background rate, is based on the assumption that a population of accelerated $\alpha$-particles with a soft energy spectrum interacting with a heavy target consisting CNO-Fe produced the resulting neutrons. $\alpha$ particles that do not produce neutrons via these interactions will subsequently range out and deposit their energy in the corona via Coulomb collisions after they have been accelerated during solar activity. The fraction of these $\alpha$-particles is dependent on the density, cross section, and the non-relativistic Bethe-Bloch expression for a given elemental composition in the corona. A rough calculation yields that the $\alpha$ emissivity needed would be of the order $8 \times 10^{21}$ alphas $\mathrm{s}^{-1} \mathrm{sr}^{-1}$, yielding an energy flux imparted to the corona of $2.1 \times 10^{-6} \mathrm{ergs} \mathrm{s}^{-1} \mathrm{sr}^{-1} \mathrm{~cm}^{-2}$. The solar wind requirement for the observed plasma temperature in the corona of $2 \times 10^{6} \mathrm{~K}$ is $\sim 10^{7} \mathrm{ergs} \mathrm{cm}^{-2} \mathrm{~s}^{-1}$ [28]. Therefore, a $3 \sigma$ detection significance of 20 neutrons above background would correspond to an energy flux $\sim 13$ orders of magnitude smaller than this requirement. This provides that if SONNE were sensitive to 20 neutron counts above background over a two-week observation time, then it would be sensitive to an energy flux of this magnitude. For $1 \%$ coronal heating by particles alone, a required input energy flux of $10^{5} \mathrm{ergs} \mathrm{cm}^{-2} \mathrm{~s}^{-1}$ would be needed. This translates into $2.7 \times 10^{28}$ neutrons s $\mathrm{s} \mathrm{sr}^{-1}$, or a neutron flux of $\sim 230$ counts s ${ }^{-1}$ at Solar Probe Plus at 20 solar radii. While it is not known what SONNE can expect within close proximity to the Sun, the results demonstrate that the instrument should be sensitive enough to detect quiescent solar neutrons that may contribute to coronal heating. 


\section{Conclusions \& Future}

Solar Probe Plus is an ambitious mission that has the ability to make unprecedented discoveries of our nearest star with a mission to the inner heliosphere. One of the potential discoveries is low-energy neutron emission in the low corona, providing existence of particle acceleration. This emission during times of quiet Sun, coupled with $\gamma$-ray data, could provide proof of small scale flaring and possibly explain the coronal heating problem. A small, compact and lightweight fast neutron spectrometer with $\gamma$-ray detection and measurement capabilities on-board Solar Probe Plus would have the potential to make these discoveries. The University of New Hampshire has developed a prototype instrument to assess the energy and imaging reconstruction abilities with a ${ }^{252} \mathrm{Cf} n / \gamma$-ray fission source and other standard laboratory $\mathrm{n} / \gamma$-ray emitting isotopes. A set of Monte Carlo simulations have also been developed to assess the instrument performance for measuring neutrons, $\gamma$-rays, and cosmic ray induced background neutrons. The neutron spectrometer demonstrates the ability to have sensitivity during times of flaring by suppressing the background using imaging. The $\gamma$-ray results are promising for measurements of a typical $\gamma$-ray solar flare given the size and resolution of the instrument. Additional sensitivity estimates show that SONNE should be sensitive enough to detect neutron emission during times of quiet Sun. The prototype instrument will continue to undergo laboratory testing and simulation studies to fully characterize the response. A more complete understanding of the background environment will be gained with the addition of solar energetic particle (SEP) events in the simulations to determine how they could affect a measurement.

\section{REFERENCES}

[1] The Johns Hopkins University Applied Physics Laboratory, "Feasible Mission Designs for Solar Probe Plus to Launch in 2015, 2016, 2017, or 2018," (2008). [Online]. Available: http://solarprobe.jhuapl.edu/mission/docs/20152018missions.pdf

[2] Hua, X. M., Koslovsky, B., and Lingenfelter, R. E., et al., "Angular and Energy Dependent Neutron Emission from Solar Flare Magnetic Loops,” ApJ Supp. 140, 563 (2002).

[3] Moser, M. R., Ryan, J. M., Bravar, U., Connell, J. J., Flückiger, E. O., MacKinnon, A. L., Macri, J. R., McConnell, M. L., McKibben, R. B., "SONNE: A Telescope For Imaging Solar Neutrons Below $30 \mathrm{MeV}$ In The Inner Heliosphere," Proc. $2^{\text {nd }}$ Solar Orbiter Workshop, SP 641 (2006).

[4] Solar Sentinels STDT, "Solar Sentinels: Report of the Science and Technology Definition Team," NASA/TM-2006214137 (2006). [Online]. Available: http://sentinels.gsfc.nasa.gov/SentinelsReport.pdf

[5] Solar Orbiter assessment team, "Payload Definition Document," ESA (2005). [Online]. Available: http://www.esa.int/science/solarorbiter

[6] Parker, E. N., "Nanoflares and the solar X-ray corona," ApJ 330, 474 (1988).

[7] Lin, R. P., Schwartz, R. A., and Kane, S. R., "Solar Hard X-ray Microflares," ApJ 283, 421 (1984).

[8] Porter, J. G., Moore, R. L., and Reichmann, E. J., "Microflares in the Solar Magnetic Network," ApJ 323, 380 (1987).

[9] McConnell, M.L., Bennett, K., MacKinnon, A., Miller, R., Rank, G., Ryan, J., and Schönfelder, V., "A Search for MeV Gamma-Ray Emission from the Quiet-Time Sun," Proc. $25^{\text {th }}$ Internat. Cosmic Ray Conf. 1, 13 (1997).

${ }^{[10]}$ Ryan, J. M., Bennett, K., Debrunner, H., Forrest, D., Lockwood, J., Loomis, M., McConnell, M., Morris, D., Schönfelder, V., Swanenburg, B. N., and Webber, W., "COMPTEL Measurements of Solar Flare Neutrons," Adv. Space Res., 13(9), 255 (1993).

[11] Woolf, R. S., Bloser, P. F., Bravar, U., Legere, J. S., McConnell, M. L., Macri, J. R., Mallik, P. C., Pirard, B., Ryan, J. M., and Wood, J. R., "Imaging and Spectroscopy of Fission Neutrons with the FNIT experiment," IEEE Conference on Technologies for Homeland Security Proc., 1-1 (2009).

[12] Toner, M. P., Ryan, J. M., et al., "Improved Analysis of COMPTEL Solar Neutron Data, with Application to the 15 June 1991 Flare," IAU Symposium: Recent Insights into the Physics of the Sun and Heliosphere: Highlights from SOHO and Other Space Missions, Astronomy Society of the Pacific, 203, 573 (2001).

[13] Mesytec, "MPD-4: 4 channel particle discriminator module for liquid scintillators," (2007). [Online]. Available: http://www.mesytec.com 
[14] Cecil, R. A., Anderson, B. D., Madey, R., "Improved predictions of neutron detection efficiency for hydrocarbon scintillators from $1 \mathrm{MeV}$ to about $300 \mathrm{MeV}$," Nucl. Inst. Meth. 161, 439 (1979).

[15] Saint-Gobain Crystals and Detectors, "BC-501/BC-501A/BC-519 Liquid Scintillators," (2005). [Online]. Available: http://www.detectors.saint-gobain.com

[16] Ruben, A., Hoagland, T. E., Fox, R., Kerr, P. L., Montermann, G., and Schneider, R., "A New Four Channel Pulse Shape Discriminator,” IEEE 2007 Nucl. Sci. Symp. Conf. Rec., N15-273, 681 (2007).

[17] Knoll, G. F., [Radiation Detection and Measurement], Wiley, New York, $3^{\text {rd }}$ ed., 554 (2000).

[18] Martin, R. C., Knauer, J. B., and Balo, P. A., "Production, Distribution, and Applications of Californium-252 Neutron Sources," IRRMA '99 $4^{\text {th }}$ Topical Meeting on Industrial Radiation and Radioisotope Measurement Applications (1999). [Online]. Available: http://www.osti.gov/bridge/servlets/purl/15053-AE6cnN/native/15053.pdf

[19] Povh, B., Rith, K., Scholz, C., and Zetche, F., [Particles and Nuclei: An Introduction to the Physical Concepts], Springer-Verlag, Berlin Heidelberg New York, $4^{\text {th }}$ ed., 310 (2004).

[20] Agostinelli, S., et al., "GEANT4: A Simulation Toolkit," Nucl. Instr. Meth. A 506, 250 (2003).

[21] Moser, M. R., "Theoretical Contributions to the Development of Neutron Scatter Telescopes for Fast Solar Neutron Observation in Space," University of Bern, PhD thesis, 165 (2006).

[22] Murphy, R. J., et al., "Using gamma-ray and neutron emission to determine the solar flare accelerated particle spectra and composition and the conditions within the flare magnetic loop," ApJ Supp. 168, 167 (2007).

[23] Solar Probe STDT, "Solar Probe +: Report of the Science and Technology Definition Team," NASA/TM-2008214161 (2008). [Online]. Available: http://solarprobe.gsfc.nasa.gov/SolarProbe+Web.pdf

[24] Gleeson, L. J., and Axford, W. I., "Solar Modulation of Galactic Cosmic Rays," ApJ. 154, 1011 (1968).

[25] Pirard, B., Woolf, R. S., Bravar, U., Bruillard, P. J., Flückiger, E. O., Legere, J. S., MacKinnon, A. L., Marci, J. R., Mallik, P. C., Moser, M. R., and Ryan, J. M., "Test and simulation of a fast neutron imaging telescope," Nucl. Instr. \& Meth. A. 603, 406 (2009).

[26] Mercury Messenger Website, "Gamma-Ray and Neutron Spectrometer (GRNS), MESSENGER: MErcury Surface, Space ENvironment, GEochemistry, and Ranging" (2009). [Online]. Available: http://messenger.jhuapl.edu/instruments/GRNS.html

[27] Weidenspointner, G., Harris, M. J., Sturner, S., Teegarden, B. J., and Ferguson, C., "MGGPOD: a Monte Carlo Suite for Modeling Instrumental Line and Continuum Backgrounds in Gamma-Ray Astronomy,” ApJ Supp. 156, 69 (2005).

[28] Withbroe, G. L., and Noyes, R. W., "Mass and Energy Flow in the Solar Chromosphere and Corona," Ann. Rev. Astron. Astrophys. 15, 363 (1977). 